\title{
Nano Drug Delivery Systems to Overcome Cancer Drug Resistance - A Review
}

\author{
Koushik OS ${ }^{1}$, Rao YV', Kumar $\mathbf{P}^{2}$ and Karthikeyan $\mathbf{R}^{\mathbf{3}^{*}}$ \\ ${ }^{1}$ Department of Pharmaceutical Sciences, Vignan Pharmacy College, Vadlamudi-522213, India \\ ${ }^{2}$ Faulty of Pharmaceutical Sciences, UCSI University, Kualalampur, Malaysia \\ ${ }^{3}$ Department of Biotechnology, Acharya Nagarjuna University, Nagarjuna Nagar, Guntur-522510, India
}

\begin{abstract}
An evolving form of treatment which emphases on alternative drug delivery is nanomedicine. It reduces unfavourable side effects to normal tissues and progresses the efficacy of treatment. Resistance towards cancer drug treatment is a complicated process that comprises of numerous mechanisms. To overcome these treatment complications and the major forms of drug resistance these nanomedicine provides new possibilities. These novel nanomedicines have a fast drug design, flexible and production based on genetic profiles of tumour. To overcome various forms of multi-drug resistance different nano drugs looks hopeful. As well as new prospects for cancer treatment alternative mechanisms of drug delivery and advanced designs are to proven to be worth full in overcoming, production based on genetic profiles of tumour. For overcoming cancer drug resistance, the current study aims to show the lead of nanomedicine in the medical science arena.
\end{abstract}

Keywords: Drug resistance; Mechanism; Passive targeting; Nanotechnology

\section{Introduction}

Around the world many people are affecting with one of the most life threatening disease i.e., Cancer. An important therapeutic preference mainly for primary advanced and metastatic tumours are radiation treatments and chemotherapy. Due to the efficacy of chemotherapy is significantly limited by the intrinsic and cancer cells develop resistance to antitumor drugs [1]. To suppress such resistance many approaches have been newly tested and developed [2-5], among individual patients due to the wide variations of drug resistance mechanisms, their efficacy varies considerably in different patients. Based on the molecular characteristics of tumours, we can possibly increase the therapy outcome and take us closer to an era of personalized medicine. So, the individualized selection of drug doses and targets to suppress resistance is highly significant. The objective of the current study is to describe major mechanisms of drug resistance; approaches to overcome and suppress them with emphasis on methods of treatment and personalized selection of drugs based on the individual tumours genetic profile of patient.

\section{Mechanisms of Multidrug Resistance}

Further regrowth of cancer cells occurs during the initial phase of chemotherapy and become resistant to the treatment. To overcome the resistance to the previously used drug is combined with several drugs with various mechanisms of action. [6-9]. In the late phase of chemotherapy, cancer cells become resistant not only to the drugs used previously but also for many anticancer drugs. Multidrug resistance is usually termed as cellular resistance when tumour cells treated with one anticancer drug become resistant to a whole spectrum of drugs. Resistance towards multidrug can be either intrinsic or acquired. Cancer stem cells are vital subsets of cancer cells that are notorious for intrinsic drug resistance [10-14]. These multidrug resistance cells overexpress certain stem cell markers and survive treatment with anticancer drugs. In most cases possess multidrug resistance because they initiate a growth of new tumour cells.

This progress of multidrug resistance in cancer cells as other types of adaptation to any stress includes initial and urgent stages. Initial phase of alteration arises straight after the irritant begins to act and is incomplete and the organism functions at the physiological possible of its limit. This initial stage of adaptation usually leads to the more or less pronounced damage of the cells. In disparity, urgent adaptation arises gradually as a result of repeated long-term environmental action. On the basis of repeated realization of urgent adaptation cancer cells develops adaptation to chemotherapy. Due to this a cancer cell acquires a new quality i.e. from a drug sensitive cell evolves a drug resistant cell. To avoid death by the specific drug the mechanisms used by the cell, in many instances render the cell resistance to additional drugs that the cell has never being in contact with. Two major types of resistance that we labelled as "pump" and "non-pump" resistance [15-18].

\section{Pump Resistance}

This majorly depends on membrane-bound active drug efflux pumps that expel anticancer drugs from the cytoplasm out from the cells. Today, many such drug efflux transporters have been identified [19-22]. The main drug efflux transporters include but are not limited to $\mathrm{P}$-Glycoprotein, a family of multidrug resistance-associated proteins (MRP), lung resistance proteins (LRP), breast cancer resistance proteins (BCRP), and many others. Active drug efflux systems can be divided into two families. The first family of drug efflux pumps consists of a single Trans membrane protein those effluxes drugs by using protonmotive forces. The second family includes the adenosine triphosphate (ATP)-binding cassette $(\mathrm{ABC})$ transporters. The later mechanism is very important in the development of multidrug resistance of cancer. A typical structure of the $\mathrm{ABC}$ transporters includes four or five membrane-associated do-mains $[23,24]$. The other two do-mains

*Corresponding author: Ramadoss karthikeyan, Department of Biotechnology, Acharya Nagarjuna University, Nagarjuna Nagar, Guntur-522510, India, Tel: 91-9966847127; E-mail: rkcognosy@gmail.com

Received May 26, 2016; Accepted June 09, 2016; Published June 16, 2016

Citation: Koushik OS, Rao YV, Kumar P, Karthikeyan R (2016) Nano Drug Delivery Systems to Overcome Cancer Drug Resistance - A Review. J Nanomed Nanotechnol 7: 378. doi:10.4172/2157-7439.1000378

Copyright: (c) 2016 Koushik OS, et al. This is an open-access article distributed under the terms of the Creative Commons Attribution License, which permits unrestricted use, distribution, and reproduction in any medium, provided the original author and source are credited. 
are nucleotide-binding domains, which are located in the cytoplasm. It is generally believed that the following mechanism plays a major role in the drug efflux by $\mathrm{ABC}$ pumps. An anticancer drug enters the membrane by diffusion and interacts with drug-binding domains (DBD) of the transporter formed by the transmembrane domains 6, 5, 11 , and 12 in P-glycoprotein. Such binding stimulates ATP hydrolysis at nucleotide-binding domains (NBD) of the protein. NBDs undergo hydrolysis and changes its configuration of that reduces the affinity of the drug and leads to the drug efflux. It should be stressed that the drug ought to have a relatively low molecular weight and should be internalized by cancer cells via "simple" physical diffusion in order to be pumped out from the cells by such drug efflux pumps. This is a very important requirement that allows for over-coming pump resistance of cancer cells.

\section{Non-pump Resistance}

Several mechanisms of drug resistance in cancer cells are independent from drug efflux pumps [25]. We proposed the term "non-pump" resistance to classify such mechanisms. Although, mechanisms responsible for cellular non-pump resistance can be substantially different, they, however, all are independent from the drug efflux pumps. Unlike the drug efflux pumps, non-pump resistance mechanisms do not interfere with the entry and accumulation of drugs in tumour cells. Instead, they decrease the ability of an anticancer drug to induce cell death or prevent drug-induced damage from being efficiently translated into cell death. A cancer cell possesses many processes that prevent cell death from an anticancer drug, including but not limited to the following classes: antiapoptotic drug, inactivation, anti-oxidant defences, degradation, DNA biosynthesis, replication and repair.

One of the major mechanisms of cellular non-pump drug resistance in cancer cells represents antiapoptotic defence. This system is designed to prevent transformation of a damage induced by an anticancer drug into apoptotic cell death. A huge complex of proteins is involved in cellular antiapoptotic defence. However, the BCL2 protein is a major player in this process in most cancer cells. It is generally believed that BCL2 prevents the release on cytochrome $\mathrm{c}$ from mitochondria and therefore breaks apoptotic signal finally preventing the activation of caspases and apoptosis itself [26]. Prevention of apoptosis in several types of cancer cells occurs by substantially increased cytotoxicity of the drugs and suppression of this protein after treatment with anticancer drugs [27-33]. For the suppression of non-pump resistance BCL2 and other antiapoptotic members of the BCL2 family represent attractive targets. Cancer cells have several mechanisms like enzymatic inactivation and degradation of anticancer drugs. Both in vitro and in vivo experiments showed that the activation in cancer cells of certain drug detoxification mechanisms including glutathione-S- and UDPtransferases (usually overexpressed in the liver) in response to an anticancer drug exposure [34-36], leads to a decrease in cytotoxicity and, consequently reduces the ability of the drug to induce cell death.

Many anticancer drugs induce free radicals or activate peroxidation processes inside cancer cells [37-43]. The major consequence of activated free radical process inside the cells results in the activation of lipid peroxidation a free radical chain reaction [44]. Similar oxidative damage can occur on the level of proteins and nucleic acids [45]. A hydroxyl radical attacks polyunsaturated fatty acids in biological membranes forming the lipid radicals which further induce the creation of lipid peroxy radicals in the presence of oxygen. A new molecule of fatty acid producing lipid hydro-peroxide and the next lipid radical initiating a chain reaction that damage cellular membranes by interacting with radicals. In order to protect cells from oxygen radicals a powerful antioxidant system is developed in cancerous and normal cells during their normal life cycle [46]. Antioxidant defence strategies include prevention, interception, replacement, and repair $[47,48]$. The major antioxidant systems include the catalase, superoxide dismutase, cytochromes, antioxidants, and glutathione peroxidase. This entire antioxidant system also plays a vital role in protecting cancer cells against free radicals initiated by the drug exposure.

The important mechanisms of non-pump resistance of cancer cells against the exposure to an anticancer drug are DNA replication, biosynthesis and repair. Certain anticancer drugs can induce DNA damage directly or indirectly. Such damage, if not repaired, can lead to cell death. Normal cells have a robust and well-developed system that prevents and repairs DNA damage. The same mechanisms are being used by cancer cells to limit DNA damage induced by an anticancer drug during chemotherapy. For instance, a widely used anticancer drug doxorubicin damages the cells by intercalating DNA and inhibiting macromolecular biosynthesis. The topoisomerase II complex prevents the stopping the process of replication and release of DNA double helix by stabilizing Doxorubicin. In order to compensate the damaged molecules and thereby increasing the resistance against the treatment, these resistant cancer cells can activate topoisomerases in response to doxorubicin treatment. Several other mechanisms that do not involve drug efflux pumps are activated in drug resistant cancer cells finally leading to non-pump resistance to chemotherapy. These mechanisms may include an entire system of stress proteins, interception of cell death signal and activation of cellular metabolism etc. However, these mechanisms of non-pump resistance depend on the overexpression of certain proteins. Some of these proteins are constitutive while others have been found to be induced in response to a variety of cellular stresses including chemotherapy. Such an overexpression can be detected in the samples of tumour tissues isolated from a patient.

\section{Mechanisms of Resistance to Endocrine Targeted Therapies in Breast Cancer}

Estrogen plays a critical role in the growth and proliferation of breast cancer cells through the estrogen receptor (ER). When estrogen binds to the ER, the hormone-receptor complex induces transcription and translation of some specific genes that participate in cell proliferation. Targeting and inhibiting estrogen from stimulating tumor cell growth is an effective treatment approach in ER-positive breast cancer. ER in breast cancer was regarded as the first molecular target in the development of targeted cancer therapy [49]. Several generations of selective estrogen receptor modulators (SERMs) agents have been developed, including fulvestrant, toremifene and tamoxifen, makes estrogen from binding to the ER and blocks cancer cell growth of breast. An-other group of targeted drugs inhibiting the binding of estrogen in breast cancer is Aromatase inhibitors (AIs). Three AIs, anastrozole, exemestane, and letrozole have also been approved by the FDA for the treatment of ER-positive breast cancer.

The use of endocrine targeted therapy agents alone or in combination with chemotherapy over the past decades has significantly impeded disease progression and improved survival outcomes in patients suffering from breast cancer. Unfortunately, in many cases, endocrine resistance occurs, either de novo or through acquired mechanisms during the course of the treatment. While the particular biological mechanisms of endocrine targeted therapy resistance leftovers uncertain, several abnormal cellular events have been proposed, including: loss of ER expression and function; altered expression of coactivators or co-regulators that show a serious role in ER mediated gene transcription [50]; and increased membrane ER $\alpha$ and the resulting rapid 
ligand-independent growth factor signalling cascades, such as epidermal growth factor receptor (EGFR) and insulin-like growth factor I receptor (IGF-IR) to promote tamoxifen resistance. Other aberrant mechanisms that lead to resistance include: up-regulated expression of manganese superoxide dismutase (MnSOD) that weakens ROS concentration, which is essential for cytotoxic activity; altered drug-metabolizing enzymes; and regulation of autophagy [51]. Altered expression of specific microRNAs (miRs) may also play a role in the development of endocrine targeted therapy resistance.

\section{Mechanisms of Resistance to Specific Kinase Targeted Therapies}

In cancer cell proliferation, apoptosis, as well as drug resistance, protein kinases have been considered as important mediators involved and identified as possible and gifted anticancer therapies targets. Several strategies for targeting protein kinases have been developed, including the highly attractive kinase target BCR-ABL in chronic myelogenous leukemia (CML). On chromosome 9 and gene BCR on chromosome 22 origins the CML from the reciprocal translocation of gene ABL. Therefore the BCR-ABL fusion gene is formed, which is the genetic hall-mark of this disease, known as the "Philadelphia chromosome" $(\mathrm{Ph}+)$. Originally developed as a therapy targeting the BCR-ABL protein in CML, imatinib mesylate occupies a region of the ATP-binding pocket of ABL, inhibiting activation via a closed conformation. Imatinib therapy has also been revealed to be highly effective to gastrointestinal stromal tumor (GIST), myelodysplastic/ myeloproliferative disorders [52], and systemic mastocytosis [53].

The protein kinase-targeted BCR-ABL inhibitor imatinib has revolutionized the approach to the clinical management of CML and dramatically improved patient outcome such that imatinib is recognized as the first line agent for almost all CML patients with different disease phases currently. However, the emergence of resistance to imatinib mitigates the prospect of CML treatment. A couple of mechanisms for both intrinsic and acquired resistance to imatinib have been proposed. Amplification of the may be the main reason for resistance, in which increased tyrosine kinase production exceeds the action of drug may be due to BCR-ABL gene. More importantly, the most common mechanism of The emergence of point mutations that disrupt the bind of imatinib to BCR-ABL itself is due to CML relapse in imatinib-treated patients. More than 50 kinds of point mutations have been recognized associated with imatinib-resistance to date; most commonly, a threonine-to-isoleucine substitution (T315I) in the kinase domain inhibits imatinib from binding with the ATP-binding domain. Further-more, overexpression of ATP-binding cassette (ABC) drug transporters may also contribute to insufficient intracellular drug levels of small molecule drugs to some extent. $A B C$ transporters are ATP-driven extrusion pumps that expel drugs out from the cytoplasm, which have been linked to conventional cytotoxic agents. Various types of drug efflux transporters have been identified, including but not limited to: P-glycoprotein (P-gp, encoded by the MDR1 gene, also known as $\mathrm{ABCB} 1$ ), multidrug resistance-associated protein- 1 (MRP1, also known as ABCC1), lung re-sistance protein (LRP), breast cancer resistance protein (BCRP, also known as ABCG2), and many others. Recent studies have reported that certain small molecule inhibitors (imatinib, erlotinib and nilotinib) are also the substrates of energydependent efflux pumps, such as MDR1 and BCRP.

\section{Mechanisms of Resistance to Growth Factor Receptors Related Targeted Therapies}

In the progression of different epithelial and non-epithelial tumours the overexpression and activation of certain growth factor receptors have been shown to play a vital role. ErbB family genes are dysregulated in numerous cancers and play key roles in proliferation, apoptosis, drug resistance and tumour cell growth. Mutations in EGFR and HER2 often serve as "driver" events to activate important growth factor signalling path-ways, such as the RAS-ERK and PI3K-AKT pathways in tumour cells. Currently, several compounds have been devel-oped for treatment of ErbB/HER family-activated cancers, including small molecule tyrosine-kinase inhibitors laptinib, gefitinib, and erlotinib. Antibody-based therapies, such as trastuzumab, pertuzumab, cetuximab, and panituzumab have also been applied to the treatment of a number of cancers. Trastuzumab is a monoclonal antibody approved to treat certain types of breast cancer, as well as some types of gastric or gastroesophageal junction adenocarcinoma. The mechanism by which trastuzumab acts is not completely understood, but one possibility is that it binds to the juxtam-embrane domain of HER2, down-regulating the protein, and preventing HER2 from sending growth-promoting signals. Trastuzumab has been proposed to have anti-proliferative effects as well, inducing the immune system to attack cells that express high levels of HER2 via antibody-dependent cellular toxicity (ADCC).

The success of trastuzumab treatment is challenged by occurrences of inherent or acquired resistance, which lessen the anti-proliferative effects. The majority of patients with metastatic breast cancer are initially sensitive to trastuzumab, however, develop resistance within one year of treatment, and $15 \%$ of patients unfortunately relapse under trastuzu-mab based therapy. Preclinical studies have demonstrated several possible molecular resistance mechanisms. Mutations that disrupt the binding of the antibody and the target protein may be a potential mechanism by which resistance to targeted antibodies develop. Some mutated forms of HER2 have been identified and thought to regulate the activities of the receptor ensemble, which thereby enhances tumorigenicity, invasiveness; for instance, G776 (YVMA) insertion in exon 20 of a HER2 mutant exhibits higher activity than the wild-type protein in activating downstream signaling events by forming the EGFR/HER2 heterodimer. Resistance to trastuzumab has also been found to be associ-ated with overexpression of the membraneassociated glyco-protein MUC4, IGF-IR, and activated downstream PI3K/ Akt signalling pathway. Moreover, in the presence of excess EGFR ligands (e.g. transforming growth factor- $\alpha$, TGF- $\alpha$ ) and/or HER2, the resulting heterodimers drive cells towards inhibition of apoptosis, proliferation and possibly obstruct with HER2 antagonist trastuzumab [54].

\section{Mechanisms of Resistance to Angiogenesis Targeted Therapies}

Therapies targeting angiogenesis are an important modality of modern antitumor treatment for a number of tumours, metastatic cancers in particular. To grow beyond a certain size, as well as invade and migrate, tumours must obtain adequate blood supply to obtain sufficient oxygen and nutrients for continued growth. Targeted treatment directed at anti-angiogenesis has triggered the interest of many scientists, primarily focusing on tumour-derived vascular endothelial growth factor (VEGF) and signalling targeting endothelial. Hence, a number of compounds were discovered in the past few years with encouraging results in non-small cell lung cancer (NSCLC), metastatic colorectal and kidney cancer. Bevacizumab and sunitinib are FDA-approved anti-angiogenesis drugs, which inhibit angiogenesis and cell proliferation by blocking VEGF signalling. However, recent studies in several tumour models demonstrated that treatment with angiogenesis inhibitors may initiate induction of drug resistance [55].

As a result of VEGF and VEGFR inhibition several resistancepromoting adaptive alterations arise. Distinct resistance mechanisms 
to sunitinib have been proposed on the basis of preclinical studies. Cis-mutation in the activation loop of the KIT gene may be a potential cause of sunitinib resistance in GIST. Some compensatory angiogenic factors can act as substitutes to ensure functional vessel formation and stabilization, such as epidermal growth factor (EGF), platelet-derived growth factor (PDGF). Moreover, lysosomal sequestration may also be a novel mechanism of sunitinib resistance. Anti-angiogenesis drugs may subsequently aggravate the hypoxic stroma as a result of the destruction of the tumour blood vessel network. Cancer cells are able to adapt to the cytotoxic effects of anti-angiogenesis targeted agents, thereby surviving in un favorable conditions and developing resistance. The insufficient level of oxygen in tumours is also a selection pressure for aggressive and malignant phenotypes and promotes metastasis [56].

\section{Mechanisms of Resistance to Immune System Targeted Therapies}

An essential contributor to tumour progression, drug resistance and tumour induction is the tumour micro-environment. Immune cells and molecules are essential components of the tumour microenvironment. Therapeutic approaches can stimulate the immune system to specifically target tumour cells, which induces tumourspecific immunological memory, resulting in long-term disease regression and prevention of relapse in cancer patients. B-lymphocyte antigen CD20 is an activated-glycosylated phosphoprotein, which is expressed on normal B cells, but more highly expressed in hairy cell leukemia, B-cell lymphomas, B-cell chronic lymphocytic leukemia, and melanoma cancer stem cells. Rituximab is a monoclonal antibody that recognizes and binds to CD20 to treat certain types of B cell nonHodgkin lymphoma (NHL) and chronic lymphocytic leukemia (CLL) when combined with other drugs. Many patients are highly responsive to rituximab, making it one of the most and first successful antibodies in cancer therapy [57].

Cancer cells possess many processes that prevent cell death from anticancer drugs; approximately $30-60 \%$ of NHL cells are subject to rituximab resistance. By chromosome modification worse responses to rituximab are commonly driven by an insufficient level of CD20 on the neoplastic cell surface caused (e.g. p53 mutation, 11q deletion, etc.). C-terminal deletion mutations of the CD20 gene were identified in a subset of tumour samples from NHL patients and presumed to affect antibody binding and participate in rituximab resistance. Alterations in apoptotic pathway signalling triggered by the binding of rituximab to $\mathrm{CD} 20$ could reduce tumour cells to be resistant to rituximab. It was reported that prolonged rituximab exposure led to dysregulation of Bax and Bak proteins that belong the proapoptotic Bcl-2 family. Moreover, overexpression of the MAPK pathway, PI3K pathway, or $\mathrm{NF}-\kappa \mathrm{B}$ hype $\mathrm{r}$-phosphorylation can also diminish the proapoptotic effect of rituximab. Exposure to rituximab may deplete the re-serve of complement proteins and exhaust necessary effector molecules, which plays an important role in rituximab resistance. Combining fresh frozen plasma with rituximab was demonstrated to overcome complement depletion and rituximab resistance in CLL patients. Decreased affinity of the Fc region of rituximab, low numbers of nature killer cells (NK cells) with $\gamma$-receptor, Fc or a lack of rituximab - CD20 polarization can inhibit ADCC and attenuate the efficacy of the treatment [58].

\section{Mechanisms of Resistance to Other Molecular Targeted Therapies}

Growth arrest, differentiation, and apoptosis of malignant cells are influenced by histone deacetylases (HDACs) both in vitro and in vivo investigations. HDACs are a group of enzymes that remove acetyl groups from various proteins, including those that regulate gene expression. Approximately $30 \%$ of patients with advanced mycosis fungoides and Sézary syndrome (MF/SS), as well as cutaneous T-cell lymphoma (CTCL) have shown clinical responses to the HDAC inhibitor vorinostat, which alters the acetylation of key proteins. The clinical efficacy of vorinostat has also been investigated in other leukemia and solid malignancies. However, the loss of acetylation of histones $\mathrm{H} 2 \mathrm{~A}, \mathrm{H} 2 \mathrm{~B}, \mathrm{H} 3$, and $\mathrm{H} 4$, the loss of the G2/M checkpoint activation, and the loss of caspase 3-dependent and caspase 7dependent apoptosis are shown to correlate with acquired resistance to vorinostat. High levels of phosphorylated tyrosine signal transducer and activator of transcription (pSTAT3) and nuclear localization of STAT1 in malignant T cells from MF/SS patients were also attributed to poor clinical response to vorinostat [59].

Retinoid analogues and derivatives may act as potential novel therapeutic agents due to their anti-proliferative and prodifferentiation effects in the body. All-trans retinoic acid, tretinoin is a naturally occurring retinoid that plays a significant role in regulating development of malignant various epithelial cells. The mechanism of action of tretinoin is mainly through the regulation of gene expression by nuclear retinoic acid receptors (RARs) and retinoid X receptors (RXRs), each of which contains three subtypes, $\alpha, \beta, \gamma$ and Specifically, heterodimers formed by RARs and RXRs bind with retinoic acid response elements in the promoter region of target genes. Tretinoin can bind to the RAR subunit of RAR-RXR and induce transcription. However, amino acid substitution as a result of genetic mutations in the RARa ligand binding domain and the promyelocytic leukemia (PML)-B2 domain of PML-RARahave been reported as molecular mechanisms underlying resistance to all trans retinoic acid in acute promyelocytic leukemia [60].

\section{The challenges of Multidrug Resistance}

An alternative route to diffusion of drugs into cells is through targeted nano carriers that are internalized by cells provides delivery of drugs. This approach may allow targeted carriers to bypass the activity of integral membrane proteins, known as MDR transporters, which transport a variety of anticancer drugs out of the cancer cell and produce resistance against chemotherapy [61]. More often, however, it is due to the overexpression of MDR transporters that actively pump chemotherapeutic drugs out of the cell and reduce the intracellular drug doses below lethal threshold levels. Because not all cancer cells express the MDR transporters, chemotherapy will kill only drug sensitive cells that do not or only mildly express MDR transporters, while leaving behind a small population of drug resistant cells that highly express MDR transporters. Chemotherapy may fail because residual drug-resistant cells dominate the tumour population with tumour recurrence. Among the MDR transporters, the most widely investigated proteins are: P-glycoprotein; the multidrug resistance associated proteins (MRPs), of which the most studied is the MRP1 (or ABCC1); and the breast cancer resistance protein (ABCG2). These proteins have different structures, but they share a similar function of expelling chemotherapy drugs from the cells. Several studies have demonstrated the possibility of using nanocarriers to bypass the MDR transporters. SP1049C is a non-ionic (pluronic or also known as poloxamer) block copolymer composed of a hydrophobic core and hydrophilic tail that contains doxorubicin [62-64]. In an attempt to reverse MDR, vincristine-loaded lipid nanoparticles conjugated to an anti-Pgp mAb (MRK-16), showed greater cytotoxicity in resistant human myelogenous leukaemia celllines than control non-targeted 
particles - a response attributed to the inhibition of the Pgp-mediated efflux of vincristine by MRK-16. Additional reports have addressed the challenge of MDR using polymer therapeutics, polymeric nanoparticles [65], lipid nanocapsules, and micelles within cell lines or in mouse tumour models. Combination treatments with targeted nanocarriers for selective delivery of drugs and MDR pump inhibitors will likely address some of the problems posed by resistant tumours.

\section{Overcoming Targeted Therapy Resistance via Nanotechnology}

\section{Increasing drug concentration}

Systemic drug delivery: The handiest way to overcome drug resistance of cancer cells is a growth in the attention of the drugs or use of a couple of tablets with different mechanisms of movement. But, excessive doses of chemotherapeutic marketers delivered by way of oral or parenteral routes are extremely dangerous. Whilst a drug isn't always centred mainly to most cancers cells, it may potentially set off severe adverse aspect outcomes upon wholesome tissues and cells despite the fact that a few shielding actions are taken. Consequently, this technique cannot be taken into consideration as a valuable tool to overcome multidrug resistance. So as to overcome these delinquencies of the systemic transport of high doses of anticancer pills, some procedures were advanced. these tactics are aimed at handing over higher drug concentrations in order to triumph over multidrug resistance of cancer cells include neighbourhood (topical) drug shipping and focused on of anticancer tablets to tumours.

Local (topical) drug delivery: Local drug delivery has a wonderful gain over the systemic delivery. When a drug is delivered directly to the site of action, its local con-centration in targeted cells increases while the adverse side effects generally are limited. However, the shipping of the drug locally to tumours represents tremendous problems except the tumour is quite simply accessed from the outside. Unless the cancer is located on the skin or accessible organs, achievement of this task is difficult. One prospective organ suitable for a local delivery of anticancer therapeutics is the lung. For a long time the lungs were used for a systemic and local delivery of therapeutics. One of the oldest examples of respiratory administration for systemic drug delivery is inhalation anaesthesia. An increasing variety of drugs are being administered by this route to obtain a direct effect on the target tissues of the respiratory system, including corticosteroids, mast cell stabilizers, antibiotics, beta-agonists and antifungal and antiviral agents [66]. This route of drug administration is being used increasingly for other medications, such as vasoactive drugs for hormones, sedatives, resuscitation and antioxidants. Usage of intra-tracheal injection of anticancer drugs in order to treat lung cancer is currently only exploratory.

Recently, we investigated the efficacy of the delivery of drugs by in-halation when compared to the intravenous route of administration in order to treat lung cancer. Nanostructured lipid carriers (NLC) and liposomes were used as model carriers to deliver drugs locally into the lungs by inhalation. It should be stressed that most types of anticancer drugs and nucleic acids can be effectively delivered by inhalation using these types of nanocarriers. It was found that $40 \%$ to $60 \%$ of both NLC and liposomes were accumulated in the liver and only less than $25 \%$ of the injected dose was found in the lungs after intravenous injection. In contrast, the same carriers delivered by in-halation accumulated predominately in the lungs. In fact, around $80 \%$ of the carriers were found in the lungs after inhalation. These data support the efficiency of such types of local delivery of therapeutics in the treatment of lung diseases including lung cancer.
Some carriers that can be guided to the tumour site include the conjugation of anticancer drugs. This guidance can be achieved for instance by an external magnetic field if the drugs or other therapeutic agents are conjugated to magnetic nanoparticles [66]. However, such delivery options possess some limitations and can induce adverse side effects. In summary, in order to overcome drug resistance, high doses of anticancer drugs can be delivered locally to skin, lungs, or other accessible organs. However, for the most organs and tissues this approach is not feasible.

Passive targeting: To ensure their preferential accumulation in targeted tumours some anticancer drugs can be modified. We proposed to subdivide different approaches to targeting of anticancer and other drugs specifically to the site of action into two big clusters: passive and active targeting [67]. Active targeting is usually achieved when the drug is conjugated to a carrier together with a targeting component that redirects the carrier and associated drug to the specific cells. For a preferential accumulation of the modified drug specifically in the tumour or tumour environment, passive targeting includes modifications of drug or drug formulations other than using targeting moieties.

The oldest and most known approach for a passive targeting of anti-cancer drugs to tumours is the use of enhanced permeability and retention effect (EPR). The EPR effect was first described by Maeda and co-workers. The EPR effect is the result of the increased permeability of the tumour vascular endothelium to circulating macromolecules combined with limited lymphatic drainage from the tumour interstitium. High molecular weight substances or low molecular drugs coupled with high molecular weight carriers penetrate highly vascularized tumour tissues, are inefficiently removed by lymphatic drainage and therefore accumulate in tumours. The existence of the EPR effect was experimentally confirmed for many types of macromolecular anticancer drug delivery systems [68]. Despite the simplicity of the approach it has serious disadvantages. The accumulation of high molecular weight substances occurs only to solid tumours with developed vascularization, high tumour mass and poor lymphatic drainage. However, these types of solid tumours can be relatively easy removed by surgery. The efficiency of this type of passive targeting is low for multifocal metastatic tumours that cannot be removed using surgical procedures and requires chemotherapy. Consequently, the EPR effect cannot be effectively used for overcoming drug resistance in most tumours that badly require it.

Several other approaches have been proposed for a passive targeting of anticancer drugs specifically to tumour cells. Most of them use specific tumour conditions to release anticancer drugs from the delivery system specifically to the tumour environment or in tumour cells $[69,70]$. These conditions include but are not limited to particular $\mathrm{pH}$, existence of certain enzymes or microflora in specific organ or tumour. Two main disadvantages limit the applicability of such approaches to overcome multi drug resistance in tumour cells. First, the vast majority of these types of tumour targeting are based on the conditions (e.g. microflora in gastrointestinal tract) that are attributed to the entire affected organ not tumour itself. This passive targeting option does not protect healthy cells and tissues from the targeted organ against the destructive action of highly toxic anticancer agents. Second, targeted tumour conditions (such as $\mathrm{pH}$, or expression of certain enzymes) can often be found (although usually in less pronounced degree) in healthy organs and cells. The latter opens a door for severe adverse side effects of chemotherapy.

Nanocarriers are nano sized substances (diameter 1-100 nm) which can carry a couple of tablets and/or imaging sellers. as a result of their excessive floor-vicinity-to-quantity ratio, it's far feasible to reap 
high ligand density at the floor for targeting purposes. Nanocarriers also can be used to increase nearby drug attention by carrying the drug inside and manage-liberating it whilst sure to the objectives. Presently, herbal and synthetic polymers and lipids are normally used as drug transport vectors; the family of nanocarriers includes polymeric nanoparticles, polymer conjugates, lipid-based carriers such as micelles and liposomes, gold nanoparticles, carbon nanotubes, and dendrimers, including nano cages and nanoshells. These nanocarriers have been explored for a variety of applications such as drug delivery, imaging, photo thermal ablation of tumours, radiation sensitizers, and sentinel lymph-node mapping.

Nanomedicine formulations could passively target tumour tissues via the enhanced permeability and retention (EPR) effect [71]. Nanoparticles have a specific propensity to reach tumour cells through passive targeting due to the characteristics of tumour tissues. Functionalized nanoparticles are internalized by passive and active targeting. Active targeting is more efficient due to cells overexpressing tumour-associated proteins that allow for facilitated uptake of the functionalized nanoparticles [72]. Despite the different levels of permeability among various tumours, passive targeting pathways can be enhanced through nanocarrier modification and conjugations. Some extensively used moieties, such as lipids, cholesterol, and their derivatives, or the hydrophilic polymer polyethylene glycol (PEG), can modify the nanoparticle formulation to be more stable and resistant to degradation and clearance during extended systemic circulation halflives, enhancing bio distribution and tumour localization [73].

Up to now, at least 12 polymer-drug conjugates have entered section I and II medical trials and are specially beneficial for focused on blood vessels in tumours. Examples consist of anti-endothelial immuno conjugates, fusion proteins, and caplostatin, the primary polymer-angiogenesis inhibitor conjugates. Polymers that are chemically conjugated with drugs are frequently considered new chemical entities (NCEs) thanks to a awesome pharmacokinetic profile from. Regardless of the form of novel drug targets and complicated chemistries to be had, simplest 4 capsules (doxorubicin, camptothecin, paclitaxel, and platinate) and 4 polymers (N-(2-hydroxylpropyl) methacrylamide (HPMA) copolymer, poly-Lglutamic acid, poly (ethylene glycol) (PEG), and Dextran) were repeatedly used to expand polymer-drug conjugates.

Polymersare themostcommonly explored materialsfor constructing nanoparticle-based drug carriers. One of the earliest reports of their use for cancer therapy dates back to 1979 when adsorption of anticancer drugs to polyalkylcyanoacrylate nanoparticles was described. Couvreur et al. revealed the release mechanism of the drugs from the polymer in calf serum, followed by tissue distribution and efficacy studies in a tumour model ${ }^{63}$. This work laid the foundation for the development of doxorubicin-loaded nanoparticles that were tested in clinical trials in the mid-1980s. Polymeric nanoparticles can be made from synthetic polymers, including poly (lactic acid) (PLA) and poly(lactic co-glycolic acid), or from natural polymers such as chitosan and collagen and may be used to encapsulate drugs without chemical modification. The drugs can be released in a controlled manner through surface or bulk erosion, diffusion through the polymer matrix, swelling followed by diffusion, or in response to the local environment. Several multifunctional polymeric nanoparticles are now in various stages of pre-clinical and clinical development. Concerns arising from the use of polymer-based nanocarriers include the inherent structural heterogeneity of polymers, reflected, for example, in a high polydispersity index (the ratio of the weight-and-number-average molecular weight $\left.\left(M_{w} / M_{\mathrm{n}}\right)\right)$. There are, however, a few examples of polymeric nanoparticles that show nearhomogenous size distribution.
Lipid-based companies have attractive biological homes, such as widespread biocompatibility, biodegradability, isolation of drugs from the encircling environment, and the ability to entrap each hydrophilic and hydrophobic capsule. Through the addition of sellers to the lipid membrane or through the alteration of the surface chemistry, residences of lipid-primarily based carriers, which include their length, charge, and surface capability, can without difficulty be changed. Liposomes, polymersomes, and micelles represent a class of amphiphile-based debris. Liposomes are spherical, self-closed systems formed by way of one or numerous concentric lipid bilayers with inner aqueous stages. these days, liposomes are accepted with the aid of regulatory companies to hold a range of chemotherapeutics.

Polymersomes have a structure just like that of liposomes; however they're composed of artificial polymer amphiphiles, including PLAbased copolymers. but, as with polymer therapeutics, there are nevertheless no clinically accredited strategies that use lively mobile targeting for lipid-primarily based vendors.

Micelles, that are self-assembling closed lipid monolayers with a hydrophobic middle and hydrophilic shell, had been successfully used as pharmaceutical vendors for water-insoluble tablets. They belong to a group of amphiphilic colloids that can be shaped spontaneously underneath sure concentrations and temperatures from amphiphilic or surface-active agents (surfactants). An example of a polymeric micelle under clinical evaluation is NK911, which is a block copolymer of PEG and poly (aspartic acid). NK911, which consists of a bound doxorubicin fraction $(\sim 45 \%)$ and a free drug, was evaluated for metastatic pancreatic cancer treatment. Another carrier is NK105, a micelle containing paclitaxel, was evaluated for pancreatic, colonic and gastric tumour treatment.

Lipid-based carriers pose several challenges, which represent general issues in the use of other targeted nanocarriers such as polymeric nanoparticles. For instance, upon intravenous injection, debris are swiftly cleared from the bloodstream by the reticuloendothelial defence mechanism, no matter particle composition [74]. Moreover, instability of the provider and burst drug release, as well as non-particular uptake via the mononuclear phagocytic device (MPS), offers additional demanding situations for translating these vendors to the sanatorium.

Given their lengthy history, liposome-based totally vendors serve as a conventional instance of the demanding situations encountered inside the development of nanocarriers and the solutions that have been tried. as an instance, PEG has been used to improve circulate time with the aid of stabilizing and defensive micelles and liposomes from opsonisation - a plasma protein deposition method that alerts Kupffer cells within the liver to get rid of the carriers from stream[74].

In addition to rapid clearance, every other task is the short burst release of the chemotherapeutic pills from the liposomes. To conquer this phenomenon, doxorubicin, for instance, can be encapsulated inside the liposomal aqueous section by means of an ammonium sulphate gradient. This technique achieves a stable drug entrapment with negligible drug leakage throughout movement, even after prolonged house in the blood stream. In scientific practice, liposomal structures have shown preferential accumulation in tumours, through the EPR effect, and decreased toxicity of their shipment. However, lengthy-circulating liposomes can also cause extravasation of the drug in surprising sites. The most commonly experienced clinical toxic effect from the PEGylated liposomal doxorubicin is palmar-plantar erythrodysesthesia (PPE), additionally referred to as the hand-foot syndrome. PPE - a dermatologic toxicity response seen with excessive 
doses of many sorts of chemotherapy - can be addressed via converting the dosing and scheduling of the remedy. Different challenges going through the use of liposomes within the health facility encompass the high production cost, fast oxidation of a few phospholipids, and shortage of managed-release residences of encapsulated drugs [75].

To achieve temporal release of two drugs, polymers and phospholipids can be combined as a single delivery agent (polymer core/lipid shell). After locating at a tumour site through the EPR effect, the outer phospholipid shell releases an anti-angiogenesis agent, and the inner polymeric nanoparticle subsequently releases a chemotherapy agent in response to local hypoxia - shortage of oxygen. This strategy led to reduced toxicity and enhanced anti-metastatic effects in two different mouse tumour models, emphasizing the advantages of a mechanism-based design for targeted nanocarriers [76]

Organic nanoparticles consist of dendrimers, viral capsids and nanostructures made from biological building blocks along with proteins. Abraxane is an albumin-sure paclitaxel nanoparticle components approved via the FDA in 2005 as a $2 \mathrm{~d}$-line treatment for metastatic breast cancer. Abraxane was designed to address insolubility problems encountered with paclitaxel. Its use eliminates the need for toxic solvents like Cremophor EL (polyoxyethylated castor oil), which has been shown to limit the dose of Taxol that can be administered [74].

Nanoshells have optical resonances that can be adjusted to take in or scatter basically everywhere within the electromagnetic spectrum, including the close to infrared region (NIR, $820 \mathrm{~nm}$, four $\mathrm{W} \mathrm{cm}$ ${ }^{2}$ ), where transmission of light thru tissue is top-quality. Soaking up nanoshells are appropriate for hyperthermia-based totally therapeutics, where the nanoshells absorb radiation and warmth up the surrounding cancer tissue. Scattering nanoshells, however, are suited as comparison sellers for imaging applications. Currently, a cancer remedy changed into evolved based on absorption of NIR light by means of nanoshells, resulting in fast localized heating to selectively kill tumours implanted in mice. Tissues heated above the thermal damage threshold displayed coagulation, mobile shrinkage and lack of nuclear staining, that are signs of irreversible thermal harm, while control tissues appeared undamaged. A similar approach involves gold nano cages which might be smaller $(<50 \mathrm{~nm})$ than the nanoshells. Those gold nano cages can be constructed to generate heat in response to NIR light and as a consequence may also be beneficial in hyperthermia-primarily based therapeutics. Unlike nanoshells and nano cages, pure gold nanoparticles are exceedingly clean to synthesize and manipulate. Non-unique interactions that motive toxicity in wholesome tissues may additionally obstruct using many varieties of nanoparticles, but the usage of inorganic debris for image ablation significantly limits non-particular toxicity because light is locally directed. However, inorganic debris may not offer benefits over other kinds of nanoparticles for systemic concentrated on of character most cancers cells because they're now not biodegradable or small sufficient to be cleared effortlessly, resulting in potential accumulation within the body, which may additionally motive long-term toxicity. Carbon nanotubes have the ability to enter cells using "needle-like penetration" and deliver molecules into the cytoplasm. These nanoparticles are equipped with a large surface area providing for a number of attachment sites for potential targeting ligands, as well as an internal cavity that can contain either therapeutic or diagnostic agents. These carbon nanotubes also have electrical and thermal conductivity, which may prove to be useful in future, cancer therapy applications such as thermal ablations. The length and diameter of these nanotubes can be crucial for avoiding an inflammogenic effect, making smaller and thicker nanotubes more desirable and a focus on biodegradability necessary. Current approaches to nanotubes include the incorporation of drugs such as Doxorubicin and Paclitaxel, nucleic acids including antisense oligonucleotides and short interfering RNAs (siRNAs), and the use of nanotubes as contrast agents for imaging. To our knowledge, no clinical trials have begun using carbon nanotubes for the treatment or diagnosis of cancer, mainly because of toxicity concerns and their similarity to asbestos fibres [67].

Dendrimers are nicely described globular structures of multi-branched polymers which are characterized by means of a primary middle, branches of repeating units, and an outer layer of multivalent purposeful companies, as shown in. these functional organizations can electrostatically engage with charged polar molecules, while the hydrophobic inner cavities can encapsulate uncharged, non-polar molecules through a number of interactions. The outer functional organizations also allow for managed delivery of the drug with the aid of modifications that best release in a positive $\mathrm{pH}$ or when encountered by using specific enzymes; concentrated on molecules, which includes the RGD peptide or mAbs are also used. Similarly, covalent attachment of hydrophobic capsules which includes Doxorubicin and Paclitaxel is regularly hired $[69,72]$. Dendrimers, such as poly (glutamic acid)-b-poly (phenylalanine) copolymers, can also be self-assembled into micelles to deliver pills in their core. Multiple clinical trials are ongoing using amphiphilic diblock copolymer forming micelles to supply Paclitaxel to deal with breast, non-small cell lung cancer, and superior pancreatic cancer.

\section{Conclusion and Future Perspectives}

Drugs that target genomic alterations in human tumours have now been clinically validated as effective targeted cancer therapies and personalized medicine. However, most cancers are complex and contain multiple genetic aberrations. Importantly, the relatively rapid acquisition of resistance to targeted therapy that is observed in the majority of cases significantly limits the utility of genomic targeted therapeutics and remains a substantial challenge to the clinical management of advanced cancers. Increased expression of the therapeutic target that could not be completely inhibited by the standard regimen of reagents, the highly adapt-able activation of survival signalling pathways, and the flexible inactivation of downstream cell death signalling pathways are potential causes of tumour targeted drug resistance. Inside the case of elevated expression of the healing target, easy-characteristic nanomedicines may also provide necessary concentrations of drugs. However, if the primary drug goal remains unaltered and may be blocked absolutely, an alternative compensatory kinase, receptor, or signalling pathway becomes activated due to both an adaptive comments loop and a genetic mutation all through remedy. Therefore, novel combination strategies designed to interfere with multiple abnormalities are necessary to impact targeted drug resistance.

\section{Acknowledgement}

The authors are thankful to Vignan pharmacy college management for providing literature survey searching facility for this review article.

\section{References}

1. Rebucci M, Michiels C (2013) Molecular aspects of cancer cell resistance to chemotherapy. Biochem Pharmacol 85: 1219-1226.

2. Wu CP, Hsieh $\mathrm{CH}$, Wu YS (2011) The emergence of drug transporter-mediated multidrug resistance to cancer chemotherapy. Mol Pharm 8: 1996-2011.

3. Moulder S (2010) Intrinsic resistance to chemotherapy in breast cancer Womens Health (Lond Engl) 6: 821-830.

4. Ullah MF (2008) Cancer multidrug resistance (MDR): a major impediment to effective chemotherapy. Asian Pac J Cancer Prev 9: 1-6.

5. Pauwels EK, Erba P, Mariani G, Gomes CM (2007) Multidrug resistance in cancer: its mechanism and its modulation. Drug News Perspect 20: 371-377. 
6. Teoh D, Secord AA (2012) Antiangiogenic agents in combination with chemotherapy for the treatment of epithelial ovarian cancer. Int J Gyneco Cancer 22: 348-359.

7. Slovin S (2012) Chemotherapy and immunotherapy combination in advanced prostate cancer. Clin Adv Hematol Oncol 10: 90-100.

8. Inoue K, Narukawa M, Takeuchi M (2012) Factors affecting efficacy and safety of add-on combination chemotherapy for non-small-cell lung cancer: a literature-based pooled analysis of randomized controlled trials. Lung 190: 355-364.

9. Hodge JW, Ardiani A, Farsaci B, Kwilas AR, Gameiro SR (2012) The tipping point for combination therapy: cancer vaccines with radiation, chemotherapy, or targeted small molecule inhibitors. Semin Oncol 39: 323-339.

10. Bhattacharyya S, Khanduja KL (2010) New hope in the horizon: cancer stem cells. Acta Biochim Biophys Sin (Shanghai) 42: 237-242.

11. Dean $M$ (2009) ABC transporters, drug resistance, and cancer stem cells. $J$ Mammary Gland Biol Neoplasia 14: 3-9.

12. Fábián A, Barok M, Vereb G, Szöllosi J (2009) Die hard: are cancer stem cells the Bruce Willises of tumor biology? Cytometry A 75: 67-74

13. Mimeault M, Mehta PP, Hauke R, Batra SK (2008) Functions of normal and malignant prostatic stem/progenitor cells in tissue regeneration and cancer progression and novel targeting therapies. Endocr Rev 29: 234-252.

14. Shah V, Taratula O, Garbuzenko OB, Taratula OR, Rodriguez-Rodriguez L, et al. (2013) Targeted nanomedicine for suppression of CD44 and simultaneous cell death induction in ovarian cancer: an optimal delivery of siRNA and anticancer drug. Clin Cancer Res.

15. Minko T, Pakunlu RI, Wang Y, Khandare JJ, Saad M (2006) New generation of liposomal drugs for cancer. Anticancer Agents Med Chem 6: 537-552.

16. Pakunlu RI, Cook TJ, Minko T (2003) Simultaneous modulation of multidrug resistance and antiapoptotic cellular defense by MDR1 and BCL-2 targeted antisense oligonu-cleotides enhances the anticancer efficacy of doxorubicin. Pharm Res 20: 351-359.

17. Pakunlu RI, Wang Y, Saad M, Khandare JJ, Starovoytov V, et al. (2006) In vitro and in vivo intracellular liposomal delivery of antisense oligonucleotides and anticancer drug. J Control Release 114: 153-162.

18. Pakunlu RI, Wang I, Tsao W, Pozharov V, Cook TJ, et al. (2004) Enhancement of the efficacy of chemotherapy for lung cancer by simultaneous suppression of mul-tidrug resistance and antiapoptotic cellular defense: novel multicomponen deliv-ery system. Cancer Res 64: 6214-6224.

19. Tiwari AK, Sodani K, Dai CL, Ashby CR Jr, Chen ZS (2011) Revisiting the ABCs of multidrug resistance in cancer chemotherapy. Curr Pharm Biotechnol 12: 570-594

20. Chen ZS, Tiwari AK (2011) Multidrug resistance proteins (MRPs/ABCCs) in cancer chemotherapy and genetic diseases. FEBS J 278: 3226-3245.

21. Noguchi K, Katayama K, Mitsuhashi J, Sugimoto $Y$ (2009) Functions of the breast cancer resistance protein (BCRP/ABCG2) in chemotherapy. Adv Drug Deliv Rev 61: 26-33.

22. Natarajan K, Xie Y, Baer MR, Ross DD (2012) Role of breast cancer resistance protein (BCRP/ABCG2) in cancer drug resistance. Biochem Pharmacol 83: 1084-1103.

23. van Veen HW, Konings WN (1998) The ABC family of multidrug transporters in microorganisms. Biochim Biophys Acta 1365: 31-36.

24. Loo TW, Clarke DM (1999) Merck Frosst Award Lecture 1998. Molecular dissection of the human multidrug resistance P-glycoprotein. Biochem Cell Biol 77: 11-23.

25. Chaudhry P, Asselin E (2009) Resistance to chemotherapy and hormone therapy in endometrial cancer. Endocr Relat Cancer 16: 363-380.

26. Nicholson DW (2000) From bench to clinic with apoptosis-based therapeutic agents. Nature 407: 810-816.

27. Dharap SS, Minko T (2003) Targeted proapoptotic LHRH-BH3 peptide. Pharm Res 20: 889-896.

28. Dharap SS, Wang Y, Chandna P, Khandare JJ, Qiu B, et al. (2005) Tumorspecific targeting of an anticancer drug delivery system by LHRH peptide. Proc Natl Acad Sci U S A 102: 12962-12967.
29. Garbuzenko OB, Saad M, Pozharov VP, Reuhl KR, Mainelis G, et al. (2010) Inhibi-tion of lung tumor growth by complex pulmonary delivery of drugs with oligonu-cleotides as suppressors of cellular resistance. Proc Natl Acad Sc 107: 10737-10742.

30. Khandare JJ, Chandna P, Wang Y, Pozharov VP, Minko T (2006) Nove polymeric prodrug with multivalent components for cancer therapy. J Pharmacol Exp Ther 317: 929-937.

31. Taratula O, Garbuzenko OB, Kirkpatrick P, Pandya I, Savla R, et al. (2009) Surface-engineered targeted $\mathrm{PPI}$ dendrimer for efficient intracellular and intratumoral siRNA delivery. J Control Release 140: 284-293.

32. Taratula A, Kuzmov M, Shah OB, Garbuzenko T, Minko et al. (2013) Nanostructured lipid carriers as multifunctional nanomedicine platform for pulmonary co-delivery of anticancer drugs and siRNA. J Control Release 171 349-357.

33. Zhang M, Garbuzenko OB, Reuhl KR, Rodriguez-Rodriguez L, Minko T (2012) Two-in-one: combined targeted chemo and gene therapy for tumor suppression and prevention of metastases. Nanomedicine (Lond) 7: 185-197.

34. Minko T, Dharap SS, Pakunlu RI, Wang Y (2004) Molecular targeting of drug delivery systems to cancer. Curr Drug Targets 5: 389-406.

35. Minko T, Kopeckova P, Kopecek J (1999) Comparison of the anticancer effect of free and HPMA copolymer-bound adriamycin in human ovarian carcinoma cells. Pharm Res 16: 986-996.

36. Minko T, Kopeckova P, Kopecek J (2000) Efficacy of the chemotherapeutic action of HPMA copolymer-bound doxorubicin in a solid tumor model of ovarian carcinoma. Int J Cancer 86: 108-117.

37. Sinha BK (1989) Free radicals in anticancer drug pharmacology. Chem Biol Interact 69: 293-317.

38. Sinha BK, Mimnaugh EG (1990) Free radicals and anticancer drug resistance: oxygen free radicals in the mechanisms of drug cytotoxicity and resistance by certain tumors. Free Radic Biol Med 8: 567-581.

39. Getoff N (2009) Vitamin free radicals and their anticancer action. Review. In Vivo 23: 599-611.

40. Jaggi AS, Singh N (2012) Mechanisms in cancer-chemotherapeutic drugs induced peripheral neuropathy. Toxicology 291: 1-9.

41. Lokeshwar BL (2011) chemically modified non-antimicrobial tetracyclines are multi-functional drugs against advanced cancers. Pharmacol Res 63: 146-150.

42. Montero AJ, Jassem J (2011) Cellular redox pathways as a therapeutic target in the treatment of cancer. Drugs 71: 1385-1396.

43. Tertil M, Jozkowicz A, Dulak J (2010) Oxidative stress in tumor angiogenesistherapeutic targets. Curr Pharm Des 16: 3877-3894.

44. Niki E, Yoshida Y, Saito Y, Noguchi N (2005) Lipid peroxidation: mechanisms, inhibition, and biological effects. Biochem Biophys Res Commun 338: 668-676

45. Yin H, Xu L, Porter NA (2011) Free radical lipid peroxidation: mechanisms and analysis. Chem Rev 111: 5944-5972.

46. Cheeseman KH (1993) Mechanisms and effects of lipid peroxidation. Mo Aspects Med 14: 191-197.

47. Amara AA (2010) The philosophy behind exo/endo/existing antioxidants and our built-in oxidant and antioxidant system. Pharmazie 65: 711-719.

48. Irshad M, Chaudhuri PS (2002) Oxidant-antioxidant system: role and significance in human body. Indian J Exp Biol 40: 1233-1239.

49. Singh S, Dash AK (2009) Paclitaxel in cancer treatment: perspectives and prospects of its delivery challenges. Crit Rev Ther Drug Carrier Syst 26: 333-372.

50. Coombes RC, Hall E, Gibson LJ, Paridaens R, Jassem J, et al. (2004) A randomized trial of exemestane after two to three years of tamoxifen therapy in postmenopausal women with primary breast cancer. $\mathrm{N}$ Engl Med 350: 1081-1092.

51. Weisberg E, Manley PW, Cowan-Jacob SW, Hochhaus A, Griffin JD (2007) Second generation inhibitors of BCR-ABL for the treatment of imatinib-resistant chronic myeloid leukaemia. Nat RevCancer 7: 345-356.

52. Gottesman MM, Fojo T, Bates SE (2002) Multidrug resistance in cancer: role of ATP-dependent transporters. Nat Rev Cancer 2: 48-58.

53. Ali S, Coombes RC (2002) Endocrine-responsive breast cancer and strategies for combating resistance. Nat Rev Cancer 2: 101-112. 
Citation: Koushik OS, Rao YV, Kumar P, Karthikeyan R (2016) Nano Drug Delivery Systems to Overcome Cancer Drug Resistance - A Review. J Nanomed Nanotechnol 7: 378. doi:10.4172/2157-7439.1000378

54. Weinberg OK, Marquez-Garban DC, Pietras RJ (2005) New approaches to reverse resistance to hormonal therapy in human breast cancer. Drug Resist Updat 8: 219-233.

55. Dowsett M, Haynes B (2003) Hormonal effects of aromatase inhibitors: focus on premenopausal effects and interaction with tamoxifen. The Journal of steroid biochemistry and molecular biology $86: 255-263$.

56. Zhou Q, Atadja P, Davidson NE (2007) Histone deacetylase inhibitor LBH589 reactivates silenced estrogen receptor alpha (ER) gene expression without loss of DNA hypermethylation. Cancer Biologyand Therapy 6: 64 .

57. Fan P, Wang J, Santen RJ, Yue W (2007) Long-term treatment with tamoxifen facilitates translocation of estrogen receptor alpha out of the nucleus and enhances its interaction with EGFR in MCF-7 breast cancer cells. Cancer Res 67: $1352-1360$

58. Arpino G, Wiechmann L, Osborne CK, Schiff R (2008) Crosstalk between the estrogen receptor and the HER tyrosine kinase receptor family: molecular mechanism and clinical implications for endocrine therapy resistance. Endocr Rev 29: 217-233.

59. Razandi M, Pedram A, Jordan VC, Fuqua S, Levin ER (2013) Tamoxifen regulates cell fate through mitochondrial estrogen receptor beta in breast cancer. Oncogene 32: 3274-3285.

60. Johnston SR (2010) New strategies in estrogen receptor-positive breast cancer. Clin Cancer Res 16: 1979-1987.

61. Samaddar, JS, Gaddy VT, Duplantier J, Thandavan SP, Shah M, et al. (2008) A role for macroautophagy in protection against 4-hydroxytamoxifen-induced cell death and the development of antiestrogen resistance. Mol Cancer Ther 7: 2977-2987.

62. Peer D, Margalit R (2006) Fluoxetine and reversal of multidrug resistance. Cancer Lett 237: 180-187.

63. Haran, G, Cohen R, Bar LK, Barenholz Y (1993) Transmembrane ammoniumsulfate gradients inliposomes produce efficient and stable entrapment of amphipathic weak bases. Biochim Biophys Acta 115: 201-215.

64. Gabizon AA, Shmeeda H, Zalipsky S (2006) Pros and cons of the liposome platform in cancer drug targeting. J Liposome Res 16: 175-183.
65. Lorusso D, Di Stefano A, Carone V, Fagotti A, Pisconti S, et al. (2007) Pegylated liposomal doxorubicin-related palmar-plantar erythrodysesthesia ('hand-foot' syndrome). Ann Oncol 18: 1159-1164.

66. Couvreur P, Vauthier C (2006) Nanotechnology: intelligent design to treat complex disease. Pharm Res 23: 1417-1450.

67. Alonso MJ (2004) Nanomedicines for overcoming biological barriers. Biomed Pharmacother 58: 168-172.

68. Matsumura Y, Maeda HA (1986) new concept for macromolecular therapeutics in cancerchemotherapy - Mechanism of tumoritropic accumulation of proteins and the antitumor agentsmancs. Cancer Res 46: 6387-6392.

69. Yuan F, Dellian M, Fukumura D, Leunig M, Berk DA, et al. (1995) Vascular permeability in a human tumor xenograft: molecular size dependence and cutoff size. Cancer Res 55: 3752-3756.

70. Torchilin VP (2005) Recent advances with liposomes as pharmaceutical carriers. Nat Rev Drug Discov 4: 145-160.

71. Hobbs SK, Monsky WL, Yuan F, Roberts WG, Griffith L, et al. (1998) Regulation of transport pathways in tumor vessels: role of tumor type and microenvironment. Proc Natl Acad Sci U S A 95: 4607-4612.

72. Jain RK (1994) Barriers to drug delivery in solid tumors. Sci Am 271: 58-65.

73. Lopes de Menezes DE, Pilarski LM, Allen TM (1998) In vitro and in vivo targeting of immunoliposomal doxorubicin to human B-cell lymphoma. Cancer Res 58: 3320-3330.

74. Park JW (2002) Anti-HER2 immunoliposomes: enhanced efficacy attributable to targeted delivery. Clin Cancer Res 8: 1172-1181.

75. Allen TM (2002) Ligand-targeted therapeutics in anticancer therapy. Nat Rev Cancer 2: 750-763.

76. Mattheolabakis G, Rigas B, Constantinides PP (2012) Nanodelivery strategies in cancer chemotherapy: biological rationale and pharmaceutical perspectives. Nanomedicine 7: 1577-1590. 